\title{
New Results for Multipoint Singular Boundary Value Problems on a Measure Chain
}

\author{
Yan Sun, ${ }^{1,2}$ Yongping Sun, ${ }^{3}$ and Patricia J. Y. Wong ${ }^{4}$ \\ ${ }^{1}$ Department of Mathematics, Shanghai Normal University, Shanghai 200234, China \\ ${ }^{2}$ School of Mathematical Sciences, Fudan University, Shanghai 200433, China \\ ${ }^{3}$ College of Electron and Information, Zhejiang University of Media and Communications, Hangzhou, Zhejiang 310018, China \\ ${ }^{4}$ School of Electrical and Electronic Engineering, Nanyang Technological University, 50 Nanyang Avenue, Singapore 639798 \\ Correspondence should be addressed to Yan Sun; ysun@shnu.edu.cn
}

Received 23 January 2014; Revised 2 June 2014; Accepted 5 June 2014; Published 14 July 2014

Academic Editor: Yonghui Xia

Copyright (c) 2014 Yan Sun et al. This is an open access article distributed under the Creative Commons Attribution License, which permits unrestricted use, distribution, and reproduction in any medium, provided the original work is properly cited.

We study the existence and uniqueness of positive solutions for a class of singular $m$-point boundary value problems of second order differential equations on a measure chain. A sharper sufficient condition for the existence and uniqueness of $C_{\mathrm{rd}}^{1}[0, T]$ positive solutions as well as $C_{\mathrm{rd}}^{1}[0, T]$ positive solutions is obtained by the technique of lower and upper solutions and the maximal principle theorem.

\section{Introduction}

In this paper, we present the existence of positive solutions for the following second order singular $m$-point boundary value problem on a measure chain:

$$
\begin{gathered}
x^{\Delta \Delta}(t)+a(t) f(t, x(t))=0, \quad 0<t<T, \\
x(T)=0, \quad x(0)=\sum_{i=1}^{m-2} \alpha_{i} x\left(\eta_{i}\right),
\end{gathered}
$$

where $0<\alpha_{i}<T, i=1, \ldots, m-2,0<\eta_{1}<\eta_{2}<$ $\cdots<\eta_{m-2}<T$ are constants and $0<\sum_{i=1}^{m-2} \alpha_{i}<T, m \geq$ 3. We assume that $0<T<+\infty$ belong to $\mathbb{T}$. We use $(0, T)$ to denote $(0, T) \cap \mathbb{T}$, and similar notations are used for other intervals. The function $a:(0, T) \rightarrow[0,+\infty)$ is rdcontinuous, $f:(0, T) \times(0,+\infty) \rightarrow[0,+\infty)$ is continuous, and $f(t, x)$ may be singular at $t=0$ and $t=T, x=0$. Observe that when $\mathbb{T}=R$ or $\mathbb{T}=Z$, the problem (1)-(2) reduces to boundary value problems of ordinary differential equations or difference equations.

The existence of positive solutions for boundary value problem on a measure chain has been paid more attentions by many researchers. Related problems on measure chains can be found in [1-9].
Recently, by making use of the Krasnosel'skii fixed point theorem, Goodrich [1] studied the existence of at least one positive solution for the following boundary value problem:

$$
\begin{gathered}
u^{\Delta \Delta}(t)+\lambda f\left(t, u^{\sigma}(t)\right)=0, \quad t \in\left(a, \sigma^{2}(b)\right), \\
u(a)=\phi(u), \quad \alpha u\left(\sigma^{2}(b)\right)=0,
\end{gathered}
$$

where the nonlocal boundary condition $\phi: \mathscr{C}_{\mathrm{rd}}\left(\left[a, \sigma^{2}(b)\right]_{\mathbb{T}}\right.$, $\mathbb{R}) \rightarrow[0,+\infty)$ is continuous.

Sun and $\mathrm{Li}$ [5] gave the existence results of the following three-point boundary value problem on time scale $\mathbb{T}$ :

$$
\begin{gathered}
x^{\Delta \nabla}(t)+a(t) f(t, x(t))=0, \quad t \in(0, T) \subset \mathbb{T}, \\
\beta x(0)-\gamma x^{\Delta}(0)=0, \quad \alpha x(\eta)=x(T),
\end{gathered}
$$

where $\beta, \gamma \geq 0, \beta+\gamma>0, \eta \in(0, \rho(t)), 0<\alpha<T / \eta$, and $d=\beta(T-\alpha \eta)+\gamma(1-\alpha)>0$.

By applying functional-type cone expansion-compression fixed point theorem, P. Wang and Y. Wang [6] established the existence of positive solutions of the following nonlinear 
boundary value problem given by the dynamic equation on time scales:

$$
\begin{gathered}
x^{\Delta \nabla}(t)+a(t) f(t, x(t))=0, \quad t \in(0, T) \subset \mathbb{T}, \\
\beta x(0)-\gamma x^{\Delta}(0)=0, \\
\alpha x(T)-\sum_{i=1}^{m-2} a_{i} x\left(\xi_{i}\right)=b, \quad m \geq 3 .
\end{gathered}
$$

By employing the Krasnosel'skii fixed point theorem, Hao et al. [2] discussed the existence of positive solutions of the following boundary value problem on a time scale:

$$
\begin{gathered}
u^{\Delta \Delta}(t)+m(t) f\left(t, u^{\sigma}(t)\right)=0, \quad t \in(a, b), \\
\alpha u(a)-\beta u^{\Delta}(a)=0, \\
\gamma u(\sigma(b))+\delta u^{\Delta}(\sigma(b))=0 .
\end{gathered}
$$

Inspired and motivated greatly by the work of $[1,2,5-$ 9], we establish the existence and uniqueness of positive solution for singular $m$-point boundary value problem (1)(2). By constructing lower and upper solutions and using the maximal theorem, we not only obtain a sharper sufficient condition for the existence and uniqueness of $C_{\mathrm{rd}}[0, T]$ positive solution, but also prove a sufficient condition for the existence of $C_{\mathrm{rd}}^{1}[0, T]$ positive solution. Our technique is different from those of [1-9] and our results naturally complement/improve their work.

We state some basic notions connected to time scales, which can be found in [9].

Definition 1. Let $\mathbb{T}$ be a time scale. For $t \in \mathbb{T}$, the forward jump operator $\sigma: \mathbb{T} \rightarrow \mathbb{T}$ is defined by

$$
\sigma(t):=\inf \{s \in \mathbb{T}: s>t\}
$$

and one defines the backward jump operator $\rho: \mathbb{T} \rightarrow \mathbb{T}$ by

$$
\rho(t):=\sup \{s \in \mathbb{T}: s<t\} \text {. }
$$

Definition 2. One says that $t$ is right-scattered if $\sigma(t)>t$, and one says that $t$ is left-scattered if $\rho(t)<t$. Points are said to be isolated if they are both right-scattered and left-scattered.

Definition 3. One says that $t$ is right-dense if $t<\sup \mathbb{T}$ and $\sigma(t)=t$, and one says that $t$ is left-dense if $t>\inf \mathbb{T}$ and $\rho(t)=t$. Points are said to be dense if they are both rightdense and left-dense. One defines the graininess function $\mu$ : $\mathbb{T} \rightarrow[0,+\infty)$ by

$$
\mu(t):=\sigma(t)-t
$$

For convenience, one lists the following conditions which will be referred to later:

\footnotetext{
$\left(\mathrm{H}_{1}\right) 0<\alpha_{i}<T, i=1, \ldots, m-2,0<\eta_{1}<\eta_{2}<\cdots<$ $\eta_{m-2}<\sigma(T)$ are constants satisfying $0<\sum_{i=1}^{m-2} \alpha_{i}<T$, $m>3$, and $0<\sum_{i=1}^{m-2} \alpha_{i} \eta_{i}<T$;
}

$\left(\mathrm{H}_{2}\right) a:(0, T) \rightarrow[0,+\infty)$ is rd-continuous, and there exists $t_{0} \in\left[\eta_{m-2}, T\right)$ such that $a\left(t_{0}\right)>0$;

$\left(\mathrm{H}_{3}\right) f:(0, T) \times(0,+\infty) \rightarrow[0,+\infty)$ is continuous, and $f(t, x)$ is nonincreasing with respect to $x$, for all $t \in$ $(0, T), a(t) f(t, \cdot) \quad \equiv \quad 0$, and $0<\int_{0}^{T} a(t) f(t, \lambda t(T-$ t)) $d t<+\infty$, for all $\lambda>0$.

\section{Preliminaries and Lemmas}

Definition 4. A function $x(t) \in C_{\mathrm{rd}}[0, T] \cap C_{\mathrm{rd}}^{2}(0, T)$ is said to be a $C_{\mathrm{rd}}[0, T]$ positive solution of the problem (1)-(2) if $x(t)$ satisfies the problem (1)-(2) and $x(t)>0, t \in(0, T)$; a $C_{\mathrm{rd}}[0, T]$ positive solution $x(t)$ of the problem (1)-(2) is said to be a $C_{\mathrm{rd}}^{1}[0, T]$ positive solution if $x^{\Delta}(0+)$ and $x^{\Delta}(T-)$ exist, and $x(t)>0, t \in[0, T]$.

Definition 5. One says that a function $\varphi(t) \in C_{\mathrm{rd}}^{2}(0, T)$ is a lower solution of the problem (1)-(2) on $[0, T]$, if $\varphi(t) \in$ $C_{\mathrm{rd}}[0, T] \cap C_{\mathrm{rd}}^{2}[0, T]$ and satisfies

$$
\begin{gathered}
\varphi^{\Delta \Delta}(t)+a(t) f(t, \varphi(t)) \geq 0, \quad t \in(0, T), \\
\varphi(T) \leq 0, \quad \varphi(0)-\sum_{i=1}^{m-2} \alpha_{i} \varphi\left(\eta_{i}\right) \leq 0 .
\end{gathered}
$$

Similarly, $\psi(t) \in C_{\mathrm{rd}}^{2}[0, T]$ is said to be an upper solution of the problem (1)-(2) on $[0, T]$, if $\psi(t) \in C_{\mathrm{rd}}[0, T] \cap C_{\mathrm{rd}}^{2}(0, T)$ and satisfies

$$
\begin{gathered}
\psi^{\Delta \Delta}(t)+a(t) f(t, \psi(t)) \leq 0, \quad t \in(0, T), \\
\psi(T) \geq 0, \quad \psi(0)-\sum_{i=1}^{m-2} \alpha_{i} \psi\left(\eta_{i}\right) \geq 0 .
\end{gathered}
$$

One says $(\varphi(t), \psi(t))$ is a couple of lower and upper solutions of the problem (1)-(2), if there exist a lower solution $\varphi(t)$ and an upper solution $\psi(t)$ of the problem (1)-(2) such that

$$
\varphi(t) \leq \psi(t), \quad t \in[0, T]
$$

Lemma 6 (maximal principle). Suppose that $\left(H_{1}\right)$ is satisfied. In addition, assume that $0<\eta_{1}<\eta_{2}<\cdots<\eta_{m-2}<\delta_{n}<T$, $n=1,2, \ldots$. Let

$$
\begin{aligned}
Q_{n}=\left\{x(t) \in C_{\mathrm{rd}}\left[0, \delta_{n}\right] \cap C_{\mathrm{rd}}^{2}\left(0, \delta_{n}\right):\right. \\
\left.\quad x\left(\delta_{n}\right) \geq 0, x(0)-\sum_{i=1}^{m-2} \alpha_{i} x\left(\eta_{i}\right) \geq 0\right\} .
\end{aligned}
$$

For $x \in Q_{n}$ such that $-x^{\Delta \Delta}(t) \geq 0$ and for $t \in(0, T)$, then $x(t) \geq 0$ for $t \in\left[0, \delta_{n}\right]$. 
Proof. For all $x \in Q_{n}$, let

$$
\begin{gathered}
-x^{\Delta \Delta}(t)=g(t), \quad t \in\left(0, \delta_{n}\right) ; \\
x(0)-\sum_{i=1}^{m-2} \alpha_{i} x\left(\eta_{i}\right)=r_{1}, \quad x\left(\delta_{n}\right)=r_{2},
\end{gathered}
$$

then $r_{1} \geq 0, r_{2} \geq 0$, and $g(t) \geq 0, t \in\left(0, \delta_{n}\right)$.

Integrating (14) from 0 to $t$, we obtain

$$
-x^{\Delta}(t)+x^{\Delta}(0)=\int_{0}^{t} g(s) \Delta s .
$$

Again integrating (16) from 0 to $t$ and exchanging integral sequence, we get

$$
\begin{aligned}
-x( & t)+x(0)+x^{\Delta}(0) t \\
= & \int_{0}^{t}(t-s) g(s) \Delta s, \quad t \in\left(0, \delta_{n}\right) \subset(0, T) \subset \mathbb{T} .
\end{aligned}
$$

From (17) and boundary condition (15), we obtain

$$
\begin{aligned}
x(t)= & \frac{1}{A_{0}}\left(\left(\left(T-\sum_{i=1}^{m-2} \alpha_{i}\right) t+\sum_{i=1}^{m-2} \alpha_{i} \eta_{i}\right) r_{2}+\left(\delta_{n}-t\right) r_{1}\right) \\
& +\frac{\delta_{n}-t}{A_{0}} \sum_{i=1}^{m-2}\left(\alpha_{i} \int_{0}^{\delta_{n}} G_{n}\left(\eta_{i}, s\right) g(s) \Delta s\right) \\
& +\int_{0}^{\delta_{n}} G_{n}(t, s) g(s) \Delta s, \quad t \in\left(0, \delta_{n}\right),
\end{aligned}
$$

where $A_{0}=\delta_{n}\left(T-\sum_{i=1}^{m-2} \alpha_{i}\right)+\sum_{i=1}^{m-2} \alpha_{i} \eta_{i}>0$ and

$$
G_{n}(t, s)=\frac{1}{\delta_{n}} \begin{cases}s\left(\delta_{n}-t\right), & 0 \leq s<t<\delta_{n}<T, \\ t\left(\delta_{n}-s\right), & 0 \leq t<s<\delta_{n}<T .\end{cases}
$$

Consequently, from (18) and the definition of $G_{n}(t, s)$, we see that $x(t) \geq 0, t \in\left[0, \delta_{n}\right]$.

Let

$E=\{x:[0, T] \longrightarrow \mathbb{R} \mid x(t)$ is a nonnegative continuous

$$
\text { function, } x^{\Delta}(t) \text { is continuous }
$$$$
\text { on }[0, T] \text {, and } x^{\Delta \Delta}(t) \text { is }
$$

right-dense continuous on $[0, T)\}$.

We will use the Banach space $(E,\|\cdot\|)$ equipped with the norm $\|x\|=\max _{t \in[0, T]}|x(t)|$. Let

$$
P=\left\{x \in E: \text { there exists a real number } m_{x},\right.
$$
such that $\left.x(t) \geq m_{x} k(t), t \in[0, T]\right\}$, where $k(t)=G(t, t)$. Then $P$ is a positive cone of $E$. Define the nonlinear operator $B$ as follows:

$$
\begin{aligned}
B x(t)= & \int_{0}^{T} G(t, s) a(s) f(s, x(s)) \Delta s \\
& +\frac{T-t}{T\left(T-\sum_{i=1}^{m-2} \alpha_{i}\right)+\sum_{i=1}^{m-2} \alpha_{i} \eta_{i}} \\
& \times \sum_{i=1}^{m-2} \alpha_{i} \int_{0}^{T} G\left(\eta_{i}, s\right) a(s) f(s, x(s)) \Delta s,
\end{aligned}
$$

which for notational simplicity will be written as

$$
\begin{aligned}
B x(t)=\int_{0}^{T} G(t, s) a(s) f(s, x(s)) \Delta s \\
\quad+A(T-t) \int_{0}^{T} G\left(\eta_{i}, s\right) a(s) f(s, x(s)) \Delta s
\end{aligned}
$$

where $\eta_{i} \in(0, T)$ with $0<\eta_{1}<\cdots<\eta_{m-2}<T$ and

$$
\begin{gathered}
A=\frac{\sum_{i=1}^{m-2} \alpha_{i}}{T\left(T-\sum_{i=1}^{m-2} \alpha_{i}\right)+\sum_{i=1}^{m-2} \alpha_{i} \eta_{i}}, \\
G(t, s)=\frac{1}{T} \begin{cases}t(T-s), & 0 \leq t \leq s \leq T, \\
s(T-t), & 0 \leq s \leq t \leq T,\end{cases} \\
G\left(\eta_{i}, s\right)=\frac{1}{T} \begin{cases}\eta_{i}(T-s), & 0 \leq \eta_{i} \leq s \leq T, \\
s\left(T-\eta_{i}\right), & 0 \leq s \leq \eta_{i} \leq T .\end{cases}
\end{gathered}
$$

It is easy to see that

$$
\begin{gathered}
k(t)=G(t, t)=\frac{1}{T} t(T-t) \leq \frac{T}{4}, \quad t \in[0, T], \\
\frac{1}{T} k(t) k(s) \leq G(t, s) \leq k(t) .
\end{gathered}
$$

It is clear that the existence of a positive solution of (1)-(2) is equivalent to the existence of a nontrivial fixed point of $B$ in $P$.

Lemma 7. Suppose that $\left(H_{1}\right)-\left(H_{3}\right)$ hold. Then $B P \subset P$, and $B$ is a decreasing operator.

Proof. It is obvious that $k(t) \in P$, so $P$ is not empty: $\stackrel{\circ}{P} \neq \phi$. For all $x(t) \in P$, by the definition of $P$, there exists a real number $m_{x}$, such that $x(t) \geq m_{x} k(t), t \in[0, T]$. From $\left(\mathrm{H}_{3}\right)$, we know that

$$
\begin{aligned}
& \int_{0}^{T} k(s) a(s) f(s, x(s)) \Delta s \\
& \quad \leq \int_{0}^{T} k(s) a(s) f\left(s, m_{x} k(s)\right) \Delta s<+\infty .
\end{aligned}
$$


From the definition of $G(t, s)$ and (23), we find

$$
\begin{aligned}
B x(t)= & \int_{0}^{T} G(t, s) a(s) f(s, x(s)) \Delta s \\
& +A(T-t) \int_{0}^{T} G\left(\eta_{i}, s\right) a(s) f(s, x(s)) \Delta s \\
\leq & \int_{0}^{T} k(s) a(s) f(s, x(s)) \Delta s \\
& +A(T-t) \int_{0}^{T} k(s) a(s) f(s, x(s)) \Delta s \\
= & \frac{T}{T\left(T-t \sum_{i=1}^{m-2} \alpha_{i}+\sum_{i=1}^{m-2} \alpha_{i}\right)+\sum_{i=1}^{m-2} \eta_{i} \eta_{i}} \\
& \times \int_{0}^{T} k(s) a(s) f(s, x(s)) \Delta s \\
\leq & \frac{A\left(T^{2}+\sum_{i=1}^{m-2} \alpha_{i} \eta_{i}\right)}{\sum_{i=1}^{m-2} \alpha_{i}} \\
& \times \int_{0}^{T} k(s) a(s) f(s, x(s)) \Delta s<+\infty .
\end{aligned}
$$

Let $w=\max _{t \in[0, T]} x(t)$. From $\left(\mathrm{H}_{2}\right)$, we see $\int_{0}^{T} k(s) a(s) f(s$, $w) \Delta s>0$. Since $f(t, x)$ is continuous on $(0, T) \times(0,+\infty)$, thus

$$
\begin{aligned}
& \int_{0}^{T} k(s) a(s) f(s, x(s)) \Delta s \\
& \quad \geq \int_{0}^{T} k(s) a(s) f(s, w) \Delta s>0 .
\end{aligned}
$$

On the other hand, for all $x \in P$, by making use of (25), we obtain

$$
\begin{aligned}
B x(t)= & \int_{0}^{T} G(t, s) a(s) f(s, x(s)) \Delta s \\
& +A(T-t) \int_{0}^{T} G\left(\eta_{i}, s\right) a(s) f(s, x(s)) \Delta s \\
\geq & \frac{1}{T} k(t) \int_{0}^{T} k(s) a(s) f(s, x(s)) \Delta s \\
& +\frac{1}{T} A(T-t) k\left(\eta_{i}\right) \int_{0}^{T} k(s) a(s) f(s, x(s)) \Delta s \\
\geq & \frac{1}{T}\left(1+\frac{\sum_{i=1}^{m-2} \alpha_{i} \eta_{i}\left(T-\eta_{i}\right)}{T\left(T-\sum_{i=1}^{m-2} \alpha_{i}\right)+\sum_{i=1}^{m-2} \alpha_{i} \eta_{i}}\right) \\
& \times \int_{0}^{T} k(s) a(s) f(s, x(s)) \Delta s \cdot k(t)=m_{B x} k(t),
\end{aligned}
$$$$
\forall t \in[0, T],
$$

where

$$
\begin{aligned}
m_{B x}= & \frac{1}{T}\left(1+\frac{\sum_{i=1}^{m-2} \alpha_{i} \eta_{i}\left(T-\eta_{i}\right)}{T\left(T-\sum_{i=1}^{m-2} \alpha_{i}\right)+\sum_{i=1}^{m-2} \alpha_{i} \eta_{i}}\right) \\
& \times \int_{0}^{T} k(s) a(s) f(s, x(s)) \Delta s .
\end{aligned}
$$

Therefore $B x$ is well defined on $P$, and $B x \in P$ for all $x \in P$. So $B P \subset P$.

For all $x_{1}, x_{2} \in P$ with $x_{1}<x_{2}$, from $\left(\mathrm{H}_{2}\right)$, we see that

$$
\begin{aligned}
\left(B x_{1}\right)(t)= & \int_{0}^{T} G(t, s) a(s) f\left(s, x_{1}(s)\right) \Delta s \\
& +A(T-t) \int_{0}^{T} G\left(\eta_{i}, s\right) a(s) f\left(s, x_{1}(s)\right) \Delta s \\
\geq & \int_{0}^{T} G(t, s) a(s) f\left(s, x_{2}(s)\right) \Delta s \\
& +A(T-t) \int_{0}^{T} G\left(\eta_{i}, s\right) a(s) f\left(s, x_{2}(s)\right) \Delta s \\
= & \left(B x_{2}\right)(t) .
\end{aligned}
$$

Therefore, $\left(B x_{1}\right)(t) \geq\left(B x_{2}\right)(t)$ and hence $B$ is a decreasing operator.

Lemma 8. Suppose that $\left(H_{1}\right)-\left(H_{3}\right)$ hold. Then for any $x \in P$, the problem (1)-(2) has an upper solution $\bar{x}$ and a lower solution $\underline{x}$, and $(\underline{x}(t), \bar{x}(t))$ is a couple of lower and upper solution of the problem (1)-(2).

Proof. For all $x \in P$, we know that

$$
\begin{aligned}
(B x)(t)= & \int_{0}^{T} G(t, s) a(s) f(s, x(s)) \Delta s \\
& +A(T-t) \int_{0}^{T} G\left(\eta_{i}, s\right) a(s) f(s, x(s)) \Delta s \\
= & \frac{1}{T}(T-t) \int_{0}^{t} s a(s) f(s, x(s)) \Delta s \\
& +\frac{t}{T} \int_{t}^{T}(T-s) a(s) f(s, x(s)) \Delta s \\
& +A(T-t) \int_{0}^{T} G\left(\eta_{i}, s\right) a(s) f(s, x(s)) \Delta s, \\
(B x)^{\Delta}(t)= & -\frac{1}{T} \int_{0}^{t} s a(s) f(s, x(s)) \Delta s \\
& +\frac{1}{T} \int_{t}^{T}(T-s) a(s) f(s, x(s)) \Delta s \\
& -A \int_{0}^{T} G\left(\eta_{i}, s\right) a(s) f(s, x(s)) \Delta s
\end{aligned}
$$$$
(B x)^{\Delta \Delta}(t)=-a(t) f(t, x(t)), \quad t \in(0, T) .
$$ 
By simple computation, we obtain

$$
\begin{gathered}
(B x)(0)-\sum_{i=1}^{m-2} \alpha_{i}(B x)\left(\eta_{i}\right)=0, \quad(B x)(T)=0, \\
(B x)^{\Delta \Delta}(t)+a(t) f(t, x(t))=0 .
\end{gathered}
$$

Let

$$
\begin{aligned}
& \underline{x}(t)=\min \{k(t),(B k)(t)\}, \\
& \bar{x}(t)=\max \{k(t),(B k)(t)\} .
\end{aligned}
$$

Obviously $\underline{x}(t), \bar{x}(t)$ are well defined, and

$$
\underline{x}(t) \leq \bar{x}(t) .
$$

Since $B P \subset P$, then for any $k(t) \in P$, we see that $(B k)(t) \epsilon$ $P$. Thus there exists positive real number $m_{B k}$, such that $(B k)(t) \geq m_{B k} k(t)$. From (35) and (36), we know that

$$
\begin{aligned}
\bar{x}(t) \geq \underline{x}(t) & =\min \{k(t),(B k)(t)\} \\
& \geq \min \left\{1, m_{B k}\right\} k(t)=m_{1} k(t),
\end{aligned}
$$

where $m_{1}=\min \left\{1, m_{B k}\right\}$. Therefore $\underline{x}, \bar{x} \in P$. Thus $(B \underline{x})(t)$ and $(B \bar{x})(t)$ are well defined, and

$$
(B \bar{x})(t) \leq(B \underline{x})(t) \leq\left(B m_{1} k\right)(t) .
$$

Since $B$ is a nonincreasing operator, from (35), we know that

$$
\begin{aligned}
& (B \bar{x})(t) \leq(B k)(t) \leq \bar{x}(t), \\
& (B \underline{x})(t) \geq(B k)(t) \geq \underline{x}(t) .
\end{aligned}
$$

From (34) and the above discussion, we know that

$$
\begin{aligned}
(B \bar{x})^{\Delta \Delta}(t)+a(t) f(t, B \bar{x}(t)) & \\
& \geq(B \bar{x})^{\Delta \Delta}(t)+a(t) f(t, \bar{x}(t))=0, \\
(B \underline{x})^{\Delta \Delta}(t)+a(t) f(t, B \underline{x}(t)) & \\
\leq & (B \underline{x})^{\Delta \Delta}(t)+a(t) f(t, \underline{x}(t))=0 .
\end{aligned}
$$

Equation (33) implies $(B \bar{x})(t)$ and $(B \underline{x})(t)$ satisfy conditions (2). From (38) and (40), we know that

$$
(\underline{x}(t), \bar{x}(t))=((B \bar{x})(t),(B \underline{x})(t))
$$

is a couple of lower and upper solution of the problem (1)-(2), and $\bar{x}, \underline{x} \in P$. Therefore

$$
\bar{x}, \underline{x} \in C_{\mathrm{rd}}[0, T] \cap C_{\mathrm{rd}}^{2}(0, T) .
$$

Consequently

$$
\begin{gathered}
\bar{x}^{\Delta \Delta}(t)+a(t) f(t, \bar{x}(t)) \leq 0, \quad t \in(0, T) . \\
\bar{x}(T) \geq 0, \quad \bar{x}(0)-\sum_{i=1}^{m-2} \alpha_{i} \bar{x}\left(\eta_{i}\right) \geq 0 .
\end{gathered}
$$

\section{Main Results}

Theorem 9. Suppose that $\left(H_{1}\right)-\left(H_{3}\right)$ hold. Then the problem (1)-(2) has a unique positive solution $x^{*} \in C_{r d}[0, T] \cap C_{r d}^{2}(0, T)$ satisfying $x^{*}(t) \geq v k(t)$, where $v$ is a positive constant.

Proof of Theorem 9. We have the following.

(I) Existence of Positive Solution to the Problem (1)-(2). From Lemma 8, we know that the problem (1)-(2) has a couple of lower and upper solution. Let $(\phi(t), \bar{\phi}(t))$ be a couple of lower and upper solution of the problem (1)-(2). Then for any $t \in(0, T)$, we have that $k(t)>0, G(t, s), \phi(t), \bar{\phi}(t)$ are strictly positive continuous function.

Define auxiliary function $H(t, x)$ and operator $Q$ as follows:

$$
\begin{aligned}
H(t, x)= & \begin{cases}f(t, \underline{\phi}(t)), & \text { if } x<\underline{\phi}(t), \\
f(t, x), & \text { if } \underline{\phi}(t) \leq x \leq \bar{\phi}(t), \\
f(t, \bar{\phi}(t)), & \text { if } x>\bar{\phi}(t),\end{cases} \\
(Q x)(t)= & \int_{0}^{T} G(t, s) a(s) H(s, x(s)) \Delta s \\
& +A(T-t) \int_{0}^{T} G\left(\eta_{i}, s\right) a(s) H(s, x(s)) \Delta s,
\end{aligned}
$$

$\forall x \in P$.

Obviously, we see that $H(t, x):(0, T) \times(0,+\infty) \rightarrow[0,+\infty)$ is continuous.

Consider the following second order differential equation $m$-point singular boundary value problem:

$$
\begin{gathered}
y^{\Delta \Delta}(t)+a(t) H(t, y)=0, \quad t \in(0, T), \\
y(T)=0, \quad y(0)-\sum_{i=1}^{m-2} \alpha_{i} y\left(\eta_{i}\right)=0 .
\end{gathered}
$$

It is well known that the existence of a positive solution of problem (46) is equivalent to the existence of a nontrivial fixed point of $Q$ in $P$.

Now we prove that $Q$ is a completely continuous operator.

From $\phi(t) \in P$, there exists a positive real number $m_{1}$ such that $\phi(t) \geq m_{1} k(t)$, for all $t \in[0, T]$. Combining $\left(\mathrm{H}_{2}\right) \sim\left(\mathrm{H}_{3}\right)$ with (44) and (42), we know that

$$
\int_{0}^{1} k(s) a(s) f(s, \underline{\phi}(s)) \Delta s
$$


is definitely a finite real number. Denote

$$
\Gamma=\int_{0}^{T} a(s) f(s, \underline{\phi}(s)) \Delta s .
$$

Let constant $C>0$, for all $x \in \bar{P}_{c}=\{x \in P:\|x\| \leq c\}$. Thus, we obtain

$$
\begin{aligned}
(Q x)(t)= & \int_{0}^{T} G(t, s) a(s) H(s, x(s)) \Delta s \\
& +A(T-t) \int_{0}^{T} G\left(\eta_{i}, s\right) a(s) H(s, x(s)) \Delta s \\
\leq & \int_{0}^{T} k(s) a(s) H(s, \underline{\phi}(s)) \Delta s \\
& +A(T-t) \int_{0}^{T} G\left(\eta_{i}, s\right) a(s) H(s, \underline{\phi}(s)) \Delta s \\
\leq & \int_{0}^{T} k(s) a(s) H\left(s, m_{1} k(s)\right) \Delta s \\
& +A(T-t) \int_{0}^{T} k(s) a(s) H\left(s, m_{1} k(s)\right) \Delta s \\
< & +\infty .
\end{aligned}
$$

Consequently, $Q\left(\bar{P}_{c}\right)$ is uniformly bounded.

Now we prove that $Q$ is compact. Let $D \subset P$ be a bounded set. Then there exists $G_{1}>0$ such that $\|x\| \leq G_{1}$, for all $x \in$ $D$. It is easy to prove that $Q(D)$ is a bounded set in $P$. Since $G(t, s)$ is continuous on $[0, T] \times[0, T]$, thus it is uniformly continuous. Therefore, choose $0<\delta<2 \varepsilon / A \Gamma T$, for all $\varepsilon>0$, and $t^{\prime}, t^{\prime \prime}, s \in[0, T]$ such that $\left|t^{\prime}-t^{\prime \prime}\right|<\delta$; we have $\mid G\left(t_{1}, s\right)-$ $G\left(t_{2}, s\right) \mid<(\varepsilon / 2) \Gamma^{-1}$. Thus, for any $x \in D, t^{\prime}, t^{\prime \prime} \in[0, T]$ such that $\left|t^{\prime}-t^{\prime \prime}\right|<\delta$, we obtain

$$
\begin{aligned}
\left|(Q x)\left(t^{\prime}\right)-(Q x)\left(t^{\prime \prime}\right)\right| & \\
\leq & \int_{0}^{T}\left|G\left(t^{\prime}, s\right)-G\left(t^{\prime \prime}, s\right)\right| a(s) H(s, x(s)) \Delta s \\
& +A\left|t^{\prime}-t^{\prime \prime}\right| \int_{0}^{T} k(s) a(s) H(s, x(s)) \Delta s \\
< & \int_{0}^{T} \frac{\varepsilon}{2} \Gamma^{-1} a(s) H(s, x(s)) \Delta s \\
& +\frac{2 \varepsilon}{A \Gamma T} \cdot A \cdot \frac{T}{4} \cdot \int_{0}^{T} a(s) H(s, x(s)) \Delta s \\
\leq & \frac{\varepsilon}{2} \Gamma^{-1} \cdot \int_{0}^{T} a(s) f(s, \underline{\phi}(s)) \Delta s \\
& +\frac{\varepsilon}{2 \Gamma} \cdot \int_{0}^{T} a(s) f(s, \underline{\phi}(s)) \Delta s \\
< & \frac{\varepsilon}{2} \Gamma^{-1} \cdot \Gamma+\frac{\varepsilon}{2 \Gamma} \cdot \Gamma=\varepsilon .
\end{aligned}
$$

Therefore $Q(D)$ is equicontinuous on $P$. Then by making use of Arzela-Ascoli theorem [8] on time scale, we know that $Q(D)$ is relatively compact. Consequently $Q$ is compact.

On the other hand, let $\mathfrak{B}=\{x \in P: \phi(t) \leq x(t) \leq$ $\bar{\phi}(t)$, for all $t \in[0, T]\}$. Denote $M_{1}=\max _{t \in[0, T]} k(t)$. Then for any $\epsilon>0$, from (49), we know that there exist $\mu \in(0, T / 2)$ such that

$$
\begin{aligned}
& \int_{0}^{\mu} k(s) a(s) f(s, \underline{\phi}(s)) \Delta s \\
& \quad+\int_{T-\mu}^{T} k(s) a(s) f(s, \underline{\phi}(s)) \Delta s<\frac{\epsilon}{4(1+T A)} .
\end{aligned}
$$

For $R>0$, since $H(t, x)$ is continuous on $[\mu, T-\mu] \times[0, R]$, consequently uniformly continuous. Thus there exists $\mu^{\prime}>0$ satisfying $\mu>\mu^{\prime}>0$, for any $t \in[\mu, T-\mu], x_{1}, x_{2} \in[0, R]$ such that $\left|x_{1}-x_{2}\right|<\mu^{\prime}$; we have

$$
\left|a(t)\left(H\left(t, x_{1}(t)\right)-H\left(t, x_{2}(t)\right)\right)\right|<\frac{\epsilon}{2 M_{1}(1+T A)} .
$$

Thus, from (51) and (52), for all $x_{1}, x_{2} \in \mathfrak{B}$ such that $\left|x_{1}-x_{2}\right|<$ $\mu^{\prime}$, we obtain

$$
\begin{aligned}
& \left|\left(Q x_{2}\right)(t)-\left(Q x_{1}\right)(t)\right| \\
& \leq \int_{0}^{T} k(s) a(s)\left|H\left(s, x_{1}(s)\right)-H\left(s, x_{2}(s)\right)\right| \Delta s \\
& \quad+A(T-t) \int_{0}^{T} k(s) a(s)\left|H\left(s, x_{1}(s)\right)-H\left(s, x_{2}(s)\right)\right| \Delta s \\
& \leq(1+T A) M_{1} \int_{\mu}^{T-\mu} a(s)\left|H\left(s, x_{1}(s)\right)-H\left(s, x_{2}(s)\right)\right| \Delta s \\
& \quad+2(1+T A)\left(\int_{0}^{\mu} k(s) a(s) f(s, \underline{\phi}(s)) \Delta s\right. \\
& \left.\quad+\int_{T-\mu}^{T} k(s) a(s) f(s, \underline{\phi}(s)) \Delta s\right) \\
& <M_{1}(1+T A) \cdot \frac{\epsilon}{2 M_{1}(1+T A)}+2(1+T A) \cdot \frac{\epsilon}{4(1+T A)} \\
& =\epsilon
\end{aligned}
$$

which implies $Q$ is continuous on $P$. Consequently $Q$ is a completely continuous operator.

From Schauder fixed point theorem [7] on time scale, we know that $Q$ has at least one positive fixed point $\varphi \in$ $C_{\mathrm{rd}}[0, T] \cap C_{\mathrm{rd}}^{1}(0, T)$ in $P$, and $\varphi(t)$ satisfies $\varphi=Q \varphi$. Thus $\varphi(t)$ satisfies the following differential equation $m$-point boundary value problem:

$$
\begin{gathered}
\varphi^{\Delta \Delta}(t)+a(t) H(t, \varphi(t))=0, \\
\varphi(0)-\sum_{i=1}^{m-2} \alpha_{i} \varphi\left(\eta_{i}\right)=0, \quad \varphi(T)=0 .
\end{gathered}
$$


Now we will prove that $\underline{\phi}(t) \leq \varphi(t) \leq \bar{\phi}(t)$, for all $t \in$ $[0, T]$. First, we will prove that $\varphi(t) \leq \bar{\phi}(t)$, for all $t \in[0, T]$. In fact, if not, then there exists $t^{*} \in(0, T)$ such that

$$
\bar{\phi}\left(t^{*}\right)<\varphi\left(t^{*}\right) \text {. }
$$

Let $\psi=\bar{\phi}(t)-\varphi(t)$, for all $t \in[0, T]$. Denote

$$
\begin{aligned}
& \alpha=\inf \left\{t_{1}: 0 \leq t_{1}<t^{*}, \psi(t)<0, t \in\left(t_{1}, t^{*}\right)\right\}, \\
& \beta=\sup \left\{t_{2}: t^{*} \leq t_{2}<T, \psi(t)<0, t \in\left(t^{*}, t_{2}\right)\right\} .
\end{aligned}
$$

Then $\bar{\phi}(t)<\varphi(t)$, for all $t \in(\alpha, \beta)$. Thus from (44), we see that $H(t, \varphi(t))=f(t, \bar{\phi}(t))$. Combining (43) with (44), we get

$$
\begin{array}{r}
\bar{\phi}^{\Delta \Delta}(t)+a(t) H(t, \bar{\phi}(t))=\bar{\phi}^{\Delta \Delta}(t)+a(t) f(t, \bar{\phi}(t)) \leq 0 \\
\forall t \in(\alpha, \beta) .
\end{array}
$$

In view of the above discussion and (54), we have $\psi^{\Delta \Delta}(t)=$ $\bar{\phi}^{\Delta \Delta}(t)-\varphi^{\Delta \Delta}(t) \leq 0$. For above $\alpha, \beta, \psi(t)$, there are two cases: (1) $\psi(\alpha)=\psi(\beta)=0$; (2) $\psi(\alpha)>0, \psi(\beta)=0$.

If (1) holds, then $\psi(t)>0$, for all $t \in[\alpha, \beta]$ which contradicts (55).

If (2) holds, from $\psi(\alpha)>0$, we know that $\alpha=0, \psi^{\Delta}\left(t^{*}\right)=$ 0 . With the aid of $\psi^{\Delta}(t)$ increasing on $[\alpha, \beta]$ it follows that $\psi^{\Delta}(t) \geq 0, t \in\left[t^{*}, \beta\right]$, that is, $\psi(t)$ increasing on $\left[t^{*}, \beta\right]$. From $\psi(\beta)=0$ it follows that $\psi\left(t^{*}\right) \leq 0$, which contradicts (55). Thus we have $\bar{\phi}(t) \geq \varphi(t)$.

Similarly, we can verify that $\phi(t) \leq \varphi(t)$. Consequently $\varphi(t)$ is a positive solution of the problem (1)-(2).

(II) Unique Positive Solution of the Problem (1)-(2). Let $x_{1}(t), x_{2}(t)$ be two $C_{\mathrm{rd}}[0, T]$ positive solutions of the problem (1)-(2), and $x_{1}(t) \neq x_{2}(t)$. Without loss of generality, we assume that $t^{*} \in(0, T)$ such that $x_{2}\left(t^{*}\right)>x_{1}\left(t^{*}\right)$.

Let

$$
\begin{gathered}
\alpha=\inf \left\{t_{1}: 0 \leq t_{1}<t^{*}, x_{2}(t)>x_{1}(t), t \in\left(t_{1}, t^{*}\right)\right\}, \\
\beta=\sup \left\{t_{2}: t^{*} \leq t_{2} \leq T, x_{2}(t)>x_{1}(t), t \in\left(t^{*}, t_{2}\right)\right\},
\end{gathered}
$$

and $\varphi(t)=x_{2}(t)-x_{1}(t)$ for all $t \in[0, T]$. It is easy to see that there are two cases for above $\alpha, \beta, \varphi$ : (i) $\varphi(\alpha)=\varphi(\beta)=0$; (ii) $\varphi(\alpha)>0, \varphi(\beta)=0$;

From $t^{*} \in(\alpha, \beta)$, we have $x_{2}(t)>x_{1}(t), f\left(t, x_{2}(t)\right) \leq$ $f\left(t, x_{1}(t)\right)$. Thus, $\varphi^{\Delta \Delta}(t)=x_{2}^{\Delta \Delta}(t)-x_{1}^{\Delta \Delta}(t) \geq 0$ for all $t \in$ $(\alpha, \beta)$.

Case (i). From $\varphi^{\Delta \Delta}(t)>0$ and $\varphi(\alpha)=\varphi(\beta)=0$, we obtain $\varphi(t) \leq 0, t \in[\alpha, \beta]$, which contradicts $x_{2}\left(t^{*}\right)>x_{1}\left(t^{*}\right)$.

Case (ii). From $\varphi(\alpha)>0$, we know that $\alpha=0, \varphi^{\Delta}\left(t^{*}\right)=0$. With the aid of $\varphi^{\Delta}(t)$ increasing on $[\alpha, \beta]$, we get that $\varphi^{\Delta}(t) \geq$ $0, t \in\left[t^{*}, \beta\right]$; that is, $\varphi(t)$ is increasing on $\left[t^{*}, \beta\right]$. From $\varphi(\beta)=$ 0 , we see that $\varphi\left(t^{*}\right) \leq 0$, which contradicts $x_{2}\left(t^{*}\right)>x_{1}\left(t^{*}\right)$.
Therefore the problem (1)-(2) has a unique positive solution.

Theorem 10. Suppose that $\left(H_{1}\right)-\left(H_{3}\right)$ hold. In addition, one assumes that the following condition is satisfied:

$$
\begin{aligned}
\left(H_{3}^{\prime}\right) a(t) f(t, r) \not \equiv 0 \\
\quad 0<\int_{0}^{1} a(s) f(s, r k(s)) \Delta s<+\infty
\end{aligned}
$$

$$
\forall r>0 \text {. }
$$

Then, the problem (1)-(2) has a unique positive solution $\varphi \in$ $C_{r d}^{1}[0, T] \cap C_{r d}^{2}(0, T)$, and there exist positive real numbers $L>$ $l>0$ such that

$$
l(T-t) \leq \varphi(t) \leq L(T-t)
$$

Proof. By making use of Lemma 8, we know that the problem (1)-(2) has a couple of lower and upper solution. Applying Theorem 9 we see that the problem (1)-(2) has a unique positive solution $\varphi \in C_{\mathrm{rd}}^{1}[0, T] \cap C_{\mathrm{rd}}^{2}(0, T)$. From $\left(\mathrm{H}_{3}\right)$, we know that $f(t, \varphi(t))$ is integrable on $(0, T)$. Thus $\varphi^{\Delta \Delta}(t)$ is integrable on $(0, T)$. It follows from the fact that $\varphi^{\Delta}(0+)$ and $\varphi^{\Delta}(T-)$ exist that we see that $\varphi(t)$ is a $C_{\mathrm{rd}}[0, T]$ positive solution of the problem (1)-(2). Clearly, $\varphi(t)$ may be expressed by

$$
\begin{aligned}
\varphi(t)= & \int_{0}^{T} G(t, s) a(s) f(s, \varphi(s)) \Delta s \\
& +A(T-t) \int_{0}^{T} G\left(\eta_{i}, s\right) a(s) f(s, \varphi(s)) \Delta s .
\end{aligned}
$$

Thus, for any $t \in[0, T]$, we get

$$
\begin{aligned}
\varphi(t) \leq & A(T-t) \int_{0}^{T} k\left(\eta_{i}\right) a(s) f(s, \varphi(s)) \Delta s \\
& +k(t) \int_{0}^{T} a(s) f(s, \varphi(s)) \Delta s \\
\leq & \left(A k\left(\eta_{i}\right)+1\right) \int_{0}^{T} a(s) f(s, \varphi(s)) \Delta s(T-t) \\
= & L(T-t),
\end{aligned}
$$

where $L=\left(1+A k\left(\eta_{i}\right)\right) \int_{0}^{T} a(s) f(s, \varphi(s)) \Delta s$.

On the other hand,

$$
\begin{aligned}
\varphi(t) & \geq A(T-t) \int_{0}^{T} G\left(\eta_{i}, s\right) a(s) f(s, \varphi(s)) \Delta s \\
& \geq A(T-t) \min _{s \in[0, T]} G\left(\eta_{i}, s\right) \int_{0}^{T} a(s) f(s, \varphi(s)) \Delta s \\
& =l(T-t),
\end{aligned}
$$

where $l=A \min _{s \in[0, T]} G\left(\eta_{i}, s\right) \int_{0}^{T} a(s) f(s, \varphi(s)) \Delta s$. Therefore, from (62) and (63), we see that (60) holds. 
Corollary 11. Suppose that $\left(H_{1}\right)-\left(H_{3}\right)$ hold. In addition, assume that the following condition is satisfied:

$$
\left(\mathrm{H}_{4}\right) f(t, r) \not \equiv 0,0<\int_{0}^{T} k(s) f(s, r) \Delta s<+\infty \text {, for all } r>0 .
$$

Then the following problem

$$
\begin{gathered}
x^{\Delta \Delta}(t)+f(t, x)=0, \quad t \in(0, T), \\
x(T)=0, \quad x(0)-\sum_{i=1}^{m-2} \alpha_{i} x\left(\eta_{i}\right)=0
\end{gathered}
$$

has a unique positive solution $\varphi \in C_{r d}[0, T] \cap C_{r d}^{2}(0, T)$, and there exists a constant $l>0$ such that $\varphi(t) \geq l(T-t)$.

Corollary 12. Suppose that $\left(H_{1}\right)-\left(H_{3}\right)$ hold. In addition, assume that the following condition is satisfied:

$$
\left(\mathrm{H}_{5}\right) f(t, r) \not \equiv 0, \int_{0}^{T} f(s, r) \Delta s<+\infty \text {, for all } r>0 .
$$

Then the problem (64) has a unique positive solution $\psi \in$ $C_{r d}[0, T] \cap C_{r d}^{2}(0, T)$, and there exist positive constants $L>$ $l>0$ such that $l(T-t) \leq \psi(t) \leq L(T-t)$.

If $a(t)$ is nonsingular at $t=0$ or $t=T, x=0$, and for all $x \geq 0$, one has $f(t, x) \leq f(t, 0), t \in(0, T)$, then the following conclusion holds.

Theorem 13. Suppose that $a \in C_{r d}[0, T], f(t, x):[0, T] \times$ $[0,+\infty) \rightarrow[0,+\infty)$ is continuous, $a(t) f(t, r) \quad \not \equiv 0$, for all $r \geq 0$, and $f(t, x)$ is nonincreasing with respect to $x$, for all $t \in$ $[0, T]$. Then the problem (1)-(2) has a unique positive solution.

Remark 14. Under some weaker condition, we not only establish the existence of positive solution of the problem (1)(2), but also obtain the uniqueness of the positive solution.

Remark 15. Without the cavity of $f(t, x)$ and other stronger conditions imposed on $f(t, x)$, only $f(t, x)$ nonincreasing with respect to $x$, we obtain new results. The main results hold even if the problem is nonsingular.

\section{Examples}

In this section, we will present two examples to illustrate the main result in this paper.

Example 1. Let $\mathbb{T}=\{0,1 / 4,1 / 3\} \cup[1 / 2,1]$. Consider the following boundary value problem:

$$
\begin{gathered}
x^{\Delta \Delta}(t)+\frac{1}{\sqrt[3]{t(1-t) x}}=0, \quad 0<t<1, \\
x(1)=0, \quad x(0)=\frac{1}{3} x\left(\frac{1}{4}\right)+\frac{1}{6} x\left(\frac{2}{3}\right) .
\end{gathered}
$$

Then, the four-point boundary value problem (65) has at least one positive solution.

Note that $a(t)=1$, for $t \in(0,1), f(t, x)=1 / \sqrt[3]{t(1-t) x}$ is nonincreasing in $x$, and $f(t, x)$ is singular at $t=0$ and $t=1$.
Obviously, $\left(\mathrm{H}_{1}\right)$ and $\left(\mathrm{H}_{2}\right)$ are satisfied. Moreover, for any fixed $\lambda>0,\left(\mathrm{H}_{3}\right)$ follows immediately from

$$
\begin{aligned}
0 & <\int_{0}^{1} a(t) f(t, \lambda t(1-t)) d t \\
& =\int_{0}^{1} \frac{d t}{\sqrt[3]{t(1-t) \lambda t(1-t)}}<+\infty
\end{aligned}
$$

Thus, the existence of a positive solution follows from Theorem 9.

Example 2. Let $\mathbb{T}=\{0,1 / 9,1 / 7,1 / 6,1 / 5,1 / 3\} \cup[1 / 2,1]$. Consider the following boundary value problem:

$$
\begin{gathered}
x^{\Delta \Delta}(t)+\frac{t}{\sqrt[5]{t(1-t) x}}=0, \quad 0<t<1, \\
x(1)=0, \\
x(0)=\frac{1}{3} x\left(\frac{1}{6}\right)+\frac{1}{5} x\left(\frac{1}{3}\right)+\frac{1}{7} x\left(\frac{3}{4}\right)+\frac{1}{9} x\left(\frac{8}{9}\right) .
\end{gathered}
$$

Then, the four-point boundary value problem (65) has at least one positive solution.

Note that $a(t)=t$, for $t \in(0,1), f(t, x)=1 / \sqrt[5]{t(1-t) x}$ is nonincreasing in $x$, and $f(t, x)$ is singular at $t=0$ and $t=1$.

Obviously, $\left(\mathrm{H}_{1}\right)$ and $\left(\mathrm{H}_{2}\right)$ are satisfied. Moreover, for any fixed $\lambda>0,\left(\mathrm{H}_{3}\right)$ follows immediately from

$$
\begin{aligned}
0 & <\int_{0}^{1} a(t) f(t, \lambda t(1-t)) d t \\
& =\int_{0}^{1} \frac{t d t}{\sqrt[5]{t(1-t) \lambda t(1-t)}}<+\infty
\end{aligned}
$$

Thus, the existence of a positive solution follows from Theorem 9.

\section{Conflict of Interests}

The authors declare that there is no conflict of interests regarding the publication of this paper.

\section{Authors' Contribution}

All authors contributed equally to this paper. They read and approved the final paper.

\section{Acknowledgments}

The authors are very grateful to referee for his/her valuable comments and suggestions. The first author was supported financially by the Foundation of Shanghai Natural Science (13ZR1430100) and the Foundation of Shanghai Municipal Education Commission (DYL201105) and (2013M541455). The second author was supported financially by the Natural Science Foundation of Zhejiang Province of China (Y12A01012). 


\section{References}

[1] C. S. Goodrich, "Positive solutions to boundary value problems with nonlinear boundary conditions," Nonlinear Analysis: Theory, Methods \& Applications, vol. 75, no. 1, pp. 417-432, 2012.

[2] Z.-C. Hao, J. Liang, and T.-J. Xiao, "Existence results for time scale boundary value problem," Journal of Computational and Applied Mathematics, vol. 197, no. 1, pp. 156-168, 2006.

[3] Y.-H. Xia, J. Li, and P. J. Y. Wong, "On the topological classification of dynamic equations on time scales," Nonlinear Analysis: Real World Applications, vol. 14, no. 6, pp. 2231-2248, 2013.

[4] Y.-H. Xia, X. Chen, and V. G. Romanovski, "On the linearization theorem of fenner and pinto," Journal of Mathematical Analysis and Applications, vol. 400, no. 2, pp. 439-451, 2013.

[5] H.-R. Sun and W.-T. Li, "Positive solutions for nonlinear threepoint boundary value problems on time scales," Journal of Mathematical Analysis and Applications, vol. 299, no. 2, pp. 508524, 2004.

[6] P. Wang and Y. Wang, "Existence of positive solutions for second-order $m$-point boundary value problems on time scales," Acta Mathematicae Applicatae Sinica, English Series, vol. 22, no. 3, pp. 457-468, 2006.

[7] R. P. Agarwal, M. Bohner, and P. Rehak, "Half-linear dynamic equations," in Nonlinear Analysis and Applications: To V. Lakshmikantham on His 80th Birthday, pp. 1-57, Kluwer, Dodrecht, The Netherlands, 2003.

[8] V. Lakshmikantham, S. Sivasundaram, and B. Kaymakcalan, Dynamic Systems on a Measure Chain, Kluwer, Boston, Mass, USA, 1996.

[9] M. Bohner and A. Peterson, Dynamic Equations on Time Scales, Birkhäuser, Boston, Mass, USA, 2001. 


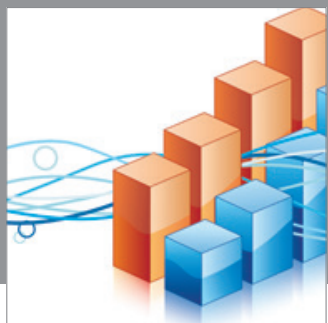

Advances in

Operations Research

mansans

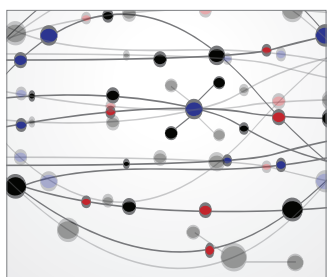

The Scientific World Journal
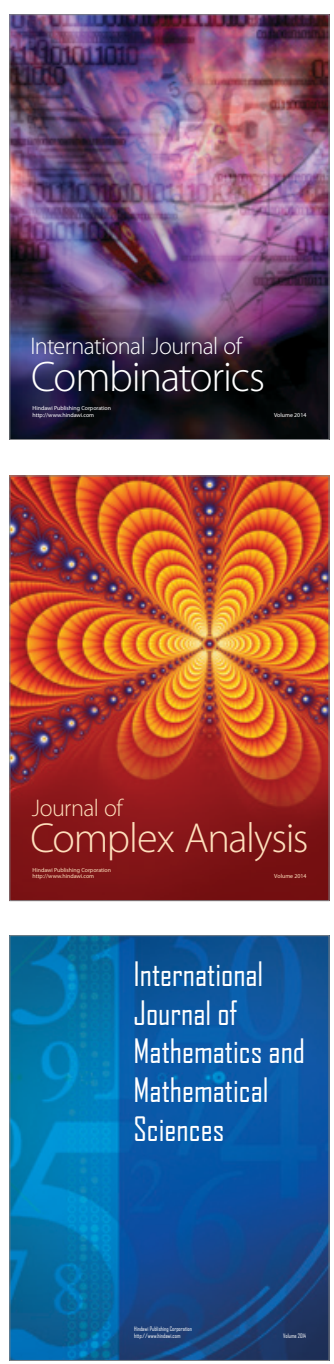
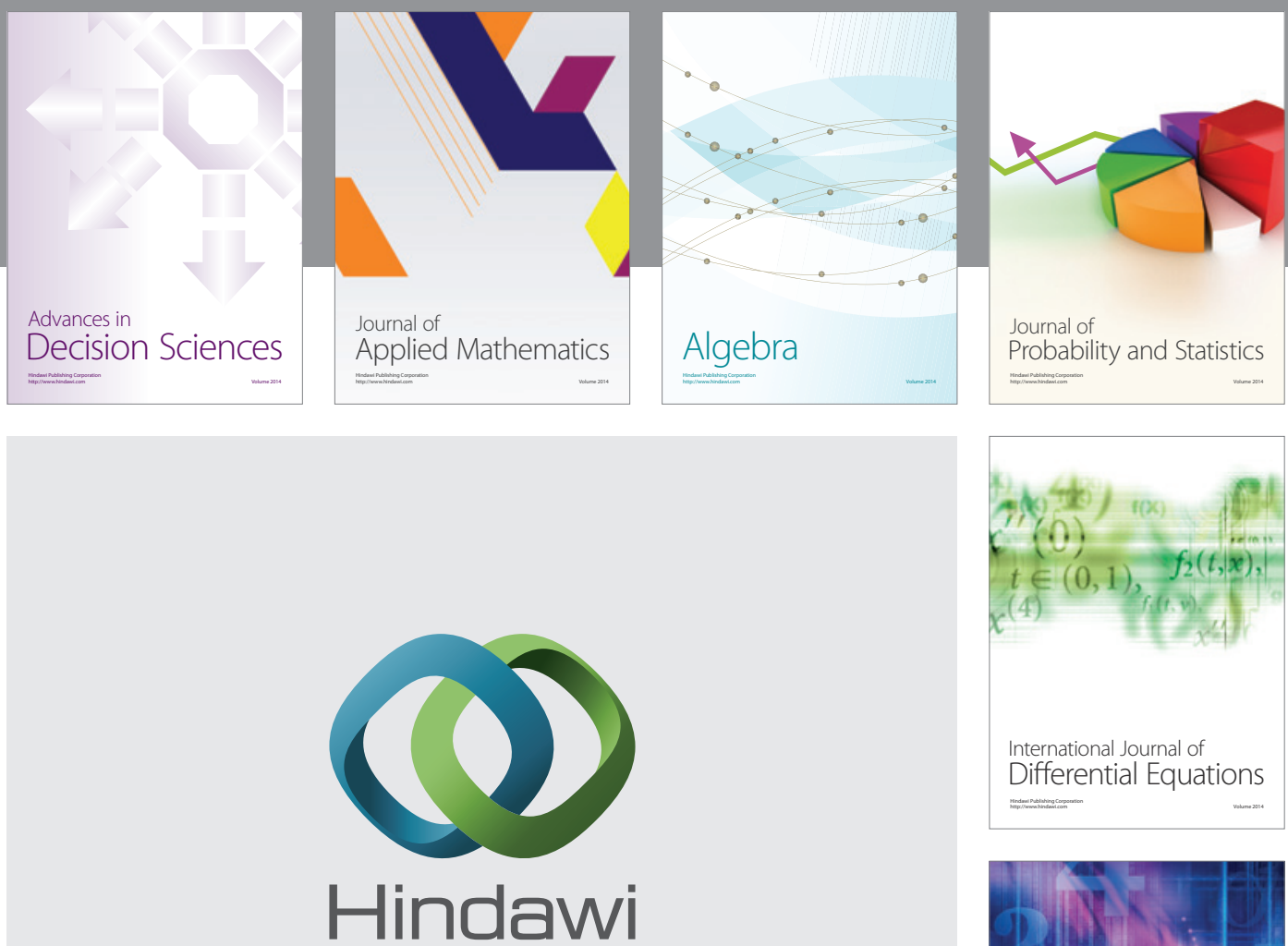

Submit your manuscripts at http://www.hindawi.com
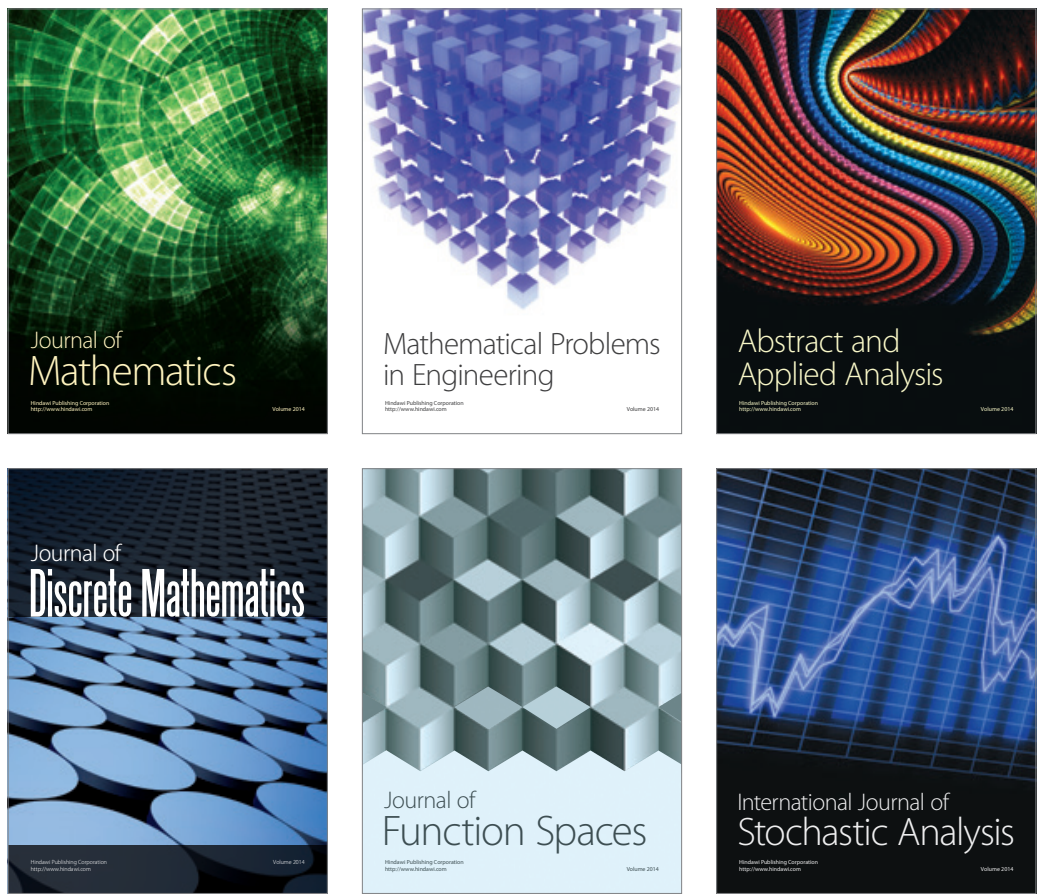

Journal of

Function Spaces

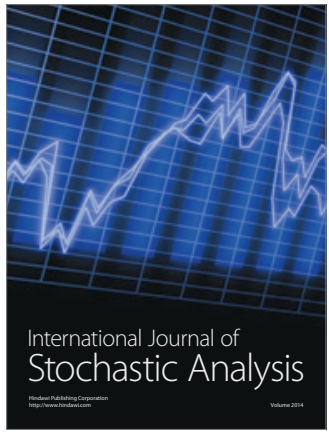

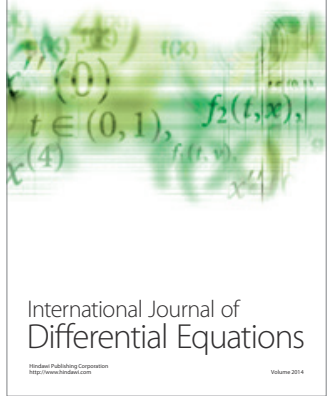
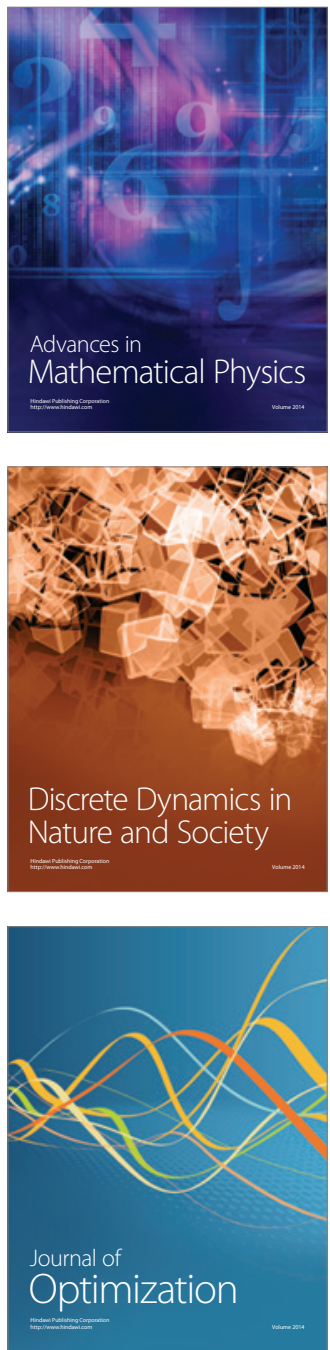